\title{
SOD2 overexpression in bone marrow-derived mesenchymal stem cells ameliorates hepatic ischemia/reperfusion injury
}

\author{
QIUYUN LI ${ }^{1}$, WEI ZHANG ${ }^{2}$ and ENHUA XIAO ${ }^{1}$ \\ ${ }^{1}$ Department of Radiology, Second Xiangya Hospital of Central South University, Changsha, Hunan 410011; \\ ${ }^{2}$ Department of Radiology, The Second People's Hospital of Hunan Province, Changsha, Hunan 410007, P.R. China
}

Received February 8, 2021; Accepted May 12, 2021

DOI: $10.3892 / \mathrm{mmr} .2021 .12310$

\begin{abstract}
Hepatic ischemia/reperfusion injury (HIRI) is a complex pathophysiological process that may develop after liver transplantation and resection surgery, as well as in uncontrolled clinical conditions. Bone marrow-derived mesenchymal stem cells (BM-MSCs) are potential targets for liver diseases. Thus, the present study aimed to investigate the effects of superoxide dismutase 2 (SOD2) overexpression in BM-MSCs on HIRI by constructing a HIRI rat model. The adenoviral vector containing SOD2 and the corresponding control vector were designed and constructed, and SOD2-overexpressing BM-MSCs were injected into the tail vein of the rats. Aspartate aminotransferase (AST) and alanine aminotransferase (ALT) levels, as well as pathological changes and the remnant liver regeneration rate were determined. The activities of SOD and glutathione peroxidase (GSH-Px), and malondialdehyde (MDA) content were measured. Reactive oxygen species (ROS) were determined with 2',7'-dichlorofluorescein diacetate and measured via fluorescence microscopy. Cell apoptosis was assessed using TUNEL staining. Moreover, the expression levels of Bax, Bcl-2 and caspase-3 were detected via western blotting. SOD2-overexpressing BM-MSCs significantly reduced the elevation of serum AST and ALT levels. Furthermore, SOD2-overexpressing BM-MSCs enhanced SOD and GSH-Px activities, and suppressed the production of MDA and ROS. Histopathological findings revealed that SOD2-overexpressing BM-MSCs decreased the number of TUNEL-positive cells in the liver. It was also found that SOD2-overexpressing BM-MSCs promoted Bcl-2 expression, but inhibited Bax and caspase-3 expression in HIRI. Collectively, these findings suggest that SOD2-overexpressing BM-MSCs may provide
\end{abstract}

Correspondence to: Professor Enhua Xiao, Department of Radiology, Second Xiangya Hospital of Central South University, 139 Renmin Road, Changsha, Hunan 410011, P.R. China

E-mail: xiaoenhua64@csu.edu.cn

Key words: hepatic ischemia/reperfusion injury, bone marrow-derived mesenchymal stem cells, superoxide dismutase 2, oxidative stress, apoptosis therapeutic support in HIRI by inhibiting oxidative stress and hepatocyte apoptosis.

\section{Introduction}

Ischemia/reperfusion (I/R) injury refers to the destruction of the normal structure and physiological function of the tissues and organs after the interruption and restoration of the blood supply (1). I/R injury often occurs when tissues and organs are traumatized, such as after thrombosis, frostbite burns, organ transplantation and surgery (2). Hepatic I/R injury (HIRI) is also a common complication after liver tissue injury and includes two stages of ischemia injury and reperfusion injury, and is associated with calcium overload, oxidative stress, cell apoptosis and metabolic acidosis $(3,4)$. However, there are currently no ideal treatments or prevention interventions that can effectively avoid HIRI.

Accumulating evidence has suggested that stem cells secrete a variety of cytokines, improve the local microenvironment, regulate the process of cell apoptosis and exert anti-inflammatory and anti-apoptotic effects, as well as promote the endogenous repair of tissues in the injured area (5-7). With the development of stem cells in the field of disease treatment, bone marrow-derived mesenchymal stem cells (BM-MSCs) have been attracting increased attention due to their wide-ranging sources, weak immunogenicity and multidirectional differentiation across germ layers $(8,9)$. After continuous subculturing and cryopreservation, BM-MSCs maintain their multidirectional differentiation ability, as well as their normal karyotype and telomerase activity, and can be induced to differentiate into hepatocytes, neuronal cells, cartilage cells and adult cells under specific conditions in vitro $(10,11)$. Moreover, BM-MSCs can attenuate injury of the brain, heart, kidney, liver and other organs, which activates strong chemical signals from damaged tissues, via differentiating into a variety of cells, secreting cytokines and chemokines, and through cell fusion (12).

A number of studies have reported that MSCs can alleviate liver histological damage, promote hepatocyte proliferation and accelerate liver tissue recovery $(13,14)$. Accumulating evidence has indicated that BM-MSC-derived hepatocyte-like exosomes reduce HIRI by enhancing autophagy (13). In a rat HIRI model, MSCs improve PTEN induced kinase 1-dependent mitochondrial-mediated apoptosis of hepatocytes 
via AMP-activated protein kinase $\alpha$ subunit activation (15). Other studies have further confirmed that allogeneic BM-MSCs can reduce HIRI by inhibiting oxidative stress and apoptosis (16). These findings indicate that BM-MSCs serve an important role in HIRI. Therefore, BM-MSCs may have potential therapeutic prospects for the treatment of HIRI.

It is worth noting that BM-MSCs can also be used as vectors to deliver protective genes by overexpressing the transfected genes in damaged parts. This not only improves the therapeutic effect, but also promotes local tissue repair (17). Superoxide dismutase 2 (SOD2) gene transfection of BM-MSCs inhibits inflammation, and it has been found that the transfected gene can be stably expressed in the body and improve glucose tolerance (18). Furthermore, upregulation of SOD2 can promote the proliferation of human umbilical cord blood-derived MSCs, and improve the oxidative stress and apoptosis of bone tissue (19). These findings suggest that BM-MSCs can be used as cell therapy, and that they are also optimal gene carriers.

Based on the aforementioned research findings, the present study aimed to establish an animal model of HIRI that closely simulated hepatic transplantation injury to investigate the protective role of SOD2-overexpressing BM-MSCs and the underlying molecular mechanisms during HIRI.

\section{Materials and methods}

Animals. All animal procedures were conducted in accordance with the International Guidelines for the Care and Use of Laboratory Animals and local ethics committee approval (20), and were also approved by the Laboratory Animal Center, The Second Xiangya Hospital of Central South University (Changsha, China). A total of 80 male Wistar rats (weight, 200-220 g; age, 8-10 weeks) were used for this study. All rats were kept in a temperature and humidity-controlled environment with a 12 -h light/dark cycle at $22-25^{\circ} \mathrm{C}$, with $50-65 \%$ humidity and free access to food and water.

Isolation of BM-MSCs. BM-MSCs were obtained using density centrifugation as previously described (18). Briefly, MSCs were flushed from the femurs and tibias of male 4-week-old Wistar rats, and were cultured with MEM (Sigma-Aldrich; Merck KGaA) supplemented with 10\% FBS (cat. no. F8687; Sigma-Aldrich; Merck KGaA), 100 U/ml penicillin and $100 \mathrm{mg} / \mathrm{l}$ streptomycin. Cultures were maintained at $37^{\circ} \mathrm{C}$ in a humidified atmosphere containing $5 \% \mathrm{CO}_{2}$. Non-adherent hematopoietic cells were removed from the adherent BM-MSCs. The culture medium was changed every 3 days. The cells were subcultured when they reached 70-80\% confluence. Third-passage BM-MSCs were used in all the experiments.

Characterization of MSCs. When the cultured cells reached $90 \%$ confluence, adherent cells were trypsinized and passaged. Cells underwent five passages before use in subsequent experiments. BM-MSCs were analyzed via antibody staining using CD90 (cat. no. SAB4700719; Sigma-Aldrich; Merck KGaA), CD105 (cat. no. MABT117; Sigma-Aldrich; Merck KGaA), CD34 (cat.no. RAB1334; Sigma-Aldrich; Merck KGaA) and CD45 (cat. no. APREST79682; Sigma-Aldrich; Merck KGaA).
MSC marker antibody staining was performed using a FACSCanto II flow cytometer (BD Biosciences).

Adenovirus production and transduction. Empty adenovirus vectors (Ad.null) and vectors encoding SOD2 (Ad.SOD2) were purchased from Shanghai Liangtai Biotechnology Company, and transductions were performed as previously described (21). To yield the adenovirus, the linearized construct DNA was transfected into 293 cells (American Type Culture Collection) using Lipofectamine ${ }^{\circledR} 2000$ (Invitrogen; Thermo Fisher Scientific, Inc.), according to the manufacturer's instructions. After $24 \mathrm{~h}$, the cells were supplied with fresh medium, and the incubation was continued for an additional 5 days. The virus was released from the cells by freezing and thawing for three consecutive cycles. After the third freeze-thaw cycle, the cells were briefly centrifuged at $4,000 \mathrm{x} \mathrm{g}$ to pellet the debris at $4^{\circ} \mathrm{C}$ for $10 \mathrm{~min}$, and the lysate was collected in sterile centrifuge tubes and stored at $-20^{\circ} \mathrm{C}$ for subsequent use. For adenovirus transduction, the MSCs were plated into 6-well plates, and the next day, the adenovirus was added into $2 \mathrm{ml}$ serum-free DMEM at a MOI of 10 . The plate was centrifuged at $220 \mathrm{x} \mathrm{g}$ for $90 \mathrm{~min}$ at $37^{\circ} \mathrm{C}$. Then, the cells were incubated in a $5 \% \mathrm{CO}_{2}$ incubator at $37^{\circ} \mathrm{C}$ for an additional $4 \mathrm{~h}$. Next, the medium was removed, and fresh complete growth medium (cat. no. 12558011; Gibco; Thermo Fisher Scientific, Inc.) was added. The cells were incubated for another $24 \mathrm{~h}$ at $37^{\circ} \mathrm{C}$ prior to analysis.

Animal model of HIRI. Rats were divided into the following groups ( $\mathrm{n}=8$ per group): Sham group, rats were subjected to laparotomy only; I/R group, rats were induced with I/R and received $\mathrm{PBS}$; +MSCs, rats were induced with $\mathrm{I} / \mathrm{R}$ and then transplanted with MSCs; and +SOD2-MSCs, rats were induced with I/R and then transplanted with SOD2-overexpressing MSCs. HIRI was induced as previously described (17). All surgical procedures were performed under anesthesia with pentobarbital sodium $(60 \mathrm{mg} / \mathrm{kg})$. A midline laparotomy was performed to expose the portal circulation, and a microaneurysm clamp was placed on the hepatic artery and portal vein to block the blood supply. This method resulted in $70 \%$ of segmental liver ischemia and prevented mesenteric veins. After $60 \mathrm{~min}$, the clamp was removed, and $1 \times 10^{6} \mathrm{PKH} 26$-labeled MSCs were immediately resuspended in $200 \mu \mathrm{l}$ PBS with a 30-gauge needle. Sham-operated rats only underwent laparotomy. After the operation, a 4/0 silk suture was used. After the animals were fully awake, they were provided with free access to food and water. During the entire procedure, the core body temperature of each rat was continuously monitored and maintained at $37.0 \pm 0.4^{\circ} \mathrm{C}$ with a heating lamp. All animals were anesthetized by intraperitoneal injection of sodium pentobarbital (30 mg/kg) until the animals lost consciousness, and then sacrificed by exsanguination.

Assessment of liver functions. To assess the severity of HIRI, the levels of serum aspartate aminotransferase (AST) and alanine aminotransferase (ALT) were determined. Rats were anesthetized with pentobarbital sodium $(60 \mathrm{mg} / \mathrm{kg})$, and $2 \mathrm{ml}$ blood was collected from the inferior vena cava with a 20-gauge needle, which was then placed in a microtainer tube with serum separator (Eppendorf), and centrifuged at $4^{\circ} \mathrm{C}$ at $3,000 \mathrm{x} \mathrm{g}$ 
for 12 min. AST (cat. no. C010-2-1; Nanjing Jiancheng Bioengineering Institute) and ALT (cat. no. C009-2-1; Nanjing Jiancheng Bioengineering Institute) levels were measured using commercial kits.

Hematoxylin and eosin $(H \& E)$ staining. Liver tissues were collected, fixed in $4 \%$ paraformaldehyde for $24 \mathrm{~h}$ at room temperature and dehydrated until transparent. The tissues were embedded in paraffin, and cut into 5- $\mu \mathrm{m}$ sections. Sections were stained with $\mathrm{H} \& \mathrm{E}$ at room temperature and observed in three random areas under a light microscope (Zeiss $\mathrm{GmbH}$ ) connected to a digital camera.

The severity of hepatic injury was evaluated in accordance with the modified Suzuki classification (22). Scores for the corresponding indicators of liver severity were determined as follows: None, 0; minimal, 1; moderate, 2; and severe, 3. For each rat, three liver sections were examined and three randomly selected high-power fields (magnification, x200) were analyzed in each section. The mean score for each animal was then determined by a summation of all scores.

Determination of SOD, glutathione peroxidase (GSH-Px) and malondialdehyde (MDA) in the liver. Liver tissues were collected and homogenized in ice-cold $0.9 \%$ saline. Following centrifugation at $3,000 \mathrm{x}$ g for $10 \mathrm{~min}$ at $4{ }^{\circ} \mathrm{C}$, the supernatant was collected and the activities of SOD (cat. no. 19160; Sigma-Aldrich; Merck KGaA), GSH-Px (cat. no. CS0260; Sigma-Aldrich; Merck KGaA) and MDA (cat. no. MAK085; Sigma-Aldrich; Merck KGaA) content were determined using commercial kits (Nanjing Jiancheng Bioengineering Institute).

Measurement of ROS. The total ROS level was measured using a ROS assay kit (cat. no. MAK143; Sigma-Aldrich; Merck KGaA), according to the manufacturer's protocol. Briefly, intracellular ROS levels were determined by measuring the oxidative conversion of cell permeable $2 \mathrm{~V}$, $7 \mathrm{~V}$-dichlorofluorescein diacetate (DCFH-DA) to fluorescent dichlorofluorescein (DCF) using a fluorospectrophotometer (Thermo Fisher Scientific, Inc.) at an excitation wavelength of $488 \mathrm{~nm}$ and an emission wavelength of $535 \mathrm{~nm}$.

Detection of apoptotic cells in liver tissues. A TUNEL kit was used to detect the apoptotic cells. Liver sections (thickness, $5 \mu \mathrm{m}$ ) were excised and fixed with $4 \%$ paraformaldehyde in PBS at room temperature for $24 \mathrm{~h}$. Fixed tissues were embedded in paraffin and stained using a TUNEL kit (cat. no. 11684795910; Roche Diagnostics), according to the manufacturer's protocol, and six sections were analyzed for each rat. The numbers of apoptotic cells and total hepatic cells in each section were counted in three randomly selected fields (magnification, $\mathrm{x} 400$ ). The apoptosis index (AI) was expressed as the mean percentage of apoptotic cells within the total number of hepatic cells for each animal.

Western blotting. A total of $100 \mathrm{mg}$ liver tissue was homogenized with lysate (cat.no. P0013; Beyotime Institute of Biotechnology), and the supernatant was centrifuged at $4,000 \times \mathrm{g}$ at $4^{\circ} \mathrm{C}$ for $10 \mathrm{~min}$. Protein quantification was performed using a BCA protein assay (Abcam) and protein samples $(20 \mu \mathrm{g})$ were collected and subjected to SDS-PAGE (10\% separation gel and $6 \%$ concentration gel). Proteins in the gel were subsequently transferred to PVDF membranes and blocked with $5 \%$ skimmed milk in TBS- $0.1 \%$ Tween- 20 at $37^{\circ} \mathrm{C}$ for $1 \mathrm{~h}$. The membranes were then incubated with the following primary antibodies: Rabbit anti-Bcl-2 (1:1,000; cat. no. SAB4500003; Sigma-Aldrich; Merck KGaA), rabbit anti-Bax (1:1,000; cat. no. SAB4502546; Sigma-Aldrich; Merck KGaA) and rabbit anti-caspase-3 (1:1,000; cat. no. C8487; Sigma-Aldrich; Merck $\mathrm{KGaA}$ ) in blocking solution at $4{ }^{\circ} \mathrm{C}$ overnight. Membranes were then washed and incubated for $5 \mathrm{~min}$ at room temperature with HRP-conjugated anti-mouse (1:5,000; cat. no. AP160P; Sigma-Aldrich; Merck KGaA) or anti-rabbit IgG secondary antibodies (1:2,000; cat. no. 31402; Invitrogen; Thermo Fisher Scientific, Inc.). The bound secondary antibodies were analyzed with an Odyssey Infrared Imaging system (LI-COR Biosciences), and proteins were normalized to $\beta$-actin (1:5,000; cat. no. SAB3500350; Sigma-Aldrich; Merck KGaA). Densitometric analysis was performed using ImageJ version 2 software (National Institutes of Health).

Reverse transcription-quantitative $(R T-q) P C R$. Total RNA was extracted using a RNeasy Mini kit (Qiagen, Inc.) from the MSCs after adenovirus transduction and purified with $75 \%$ ethanol. RNA concentration was determined by spectrophotometry. The purified total RNA (200 ng/sample) was reverse transcribed into cDNA using a transcription kit (cat. no. RR037A; Takara Bio, Inc.). qPCR reactions were performed in triplicate using a SYBR ${ }^{\circledR}$ Green Master Mix (Bio-Rad Laboratories, Inc.) and run on a LightCycler 480 system (Roche Diagnostics $\mathrm{GmbH}$ ). The following thermocycling conditions were used: Initial denaturation at $95^{\circ} \mathrm{C}$ for $30 \mathrm{sec}$; followed by 40 cycles of denaturation at $95^{\circ} \mathrm{C}$ for $5 \mathrm{sec}$, and annellation and extension at $60^{\circ} \mathrm{C}$ for $31 \mathrm{sec}$. The following primers were used in the current study: SOD2 forward, 5'-ACGTACTAGACGCGCAATT-3' and reverse, 5'-ACTTGTTAGAGTTGCGGTGG-3'; and GAPDH forward, 5'-CAGTTACTTCCCCAGCAA-3' and reverse, 5'-CACGACTCATACAGCACCT-3'. The relative gene expression was quantified using the $2^{-\Delta \Delta \mathrm{Cq}}$ method (23).

Statistical analysis. SPSS 21.0 (IBM Corp.) was used for statistical analyses. Data are presented as the mean \pm SD of experiments repeated in triplicate. For multiple comparisons, data were analyzed using one-way ANOVA followed by a Tukey's post hoc test. Analysis between two groups was performed using an unpaired Student's t-test. $\mathrm{P}<0.05$ was considered to indicate a statistically significant difference.

\section{Results}

Expression level of SOD2 in BM-MSCs with adenovirus vector transduction. To verify whether the collected cells were BM-MSCs, MSC-specific cell surface markers were detected via staining. As shown in Fig. 1A-D, BM-MSCs were positive for CD90 and CD105, which are specific MSC surface markers (24). Additionally, these cells were negative for CD34 and CD45, which are non-MSC markers. The findings suggested that these BM-MSCs were typical MSCs, and were used for subsequent experiments. 


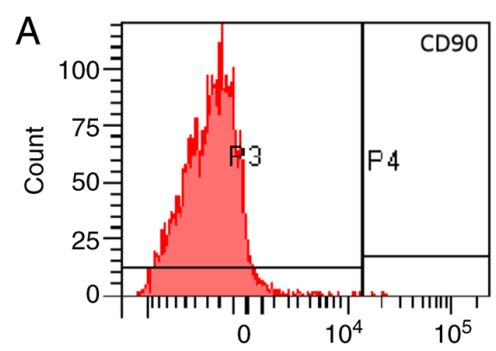

B

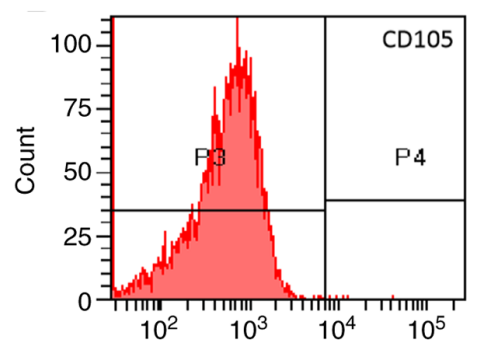

E

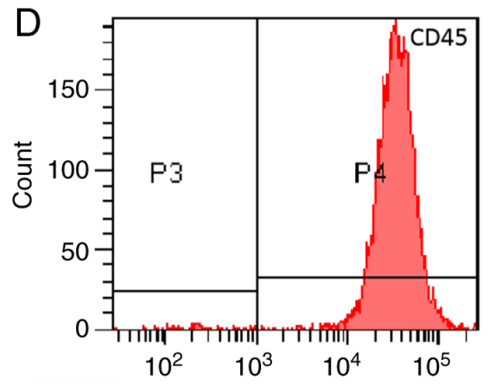

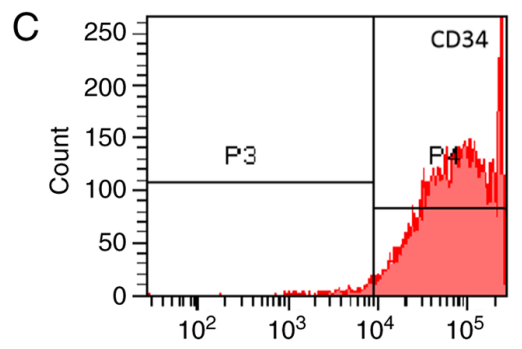

$\mathrm{F}$

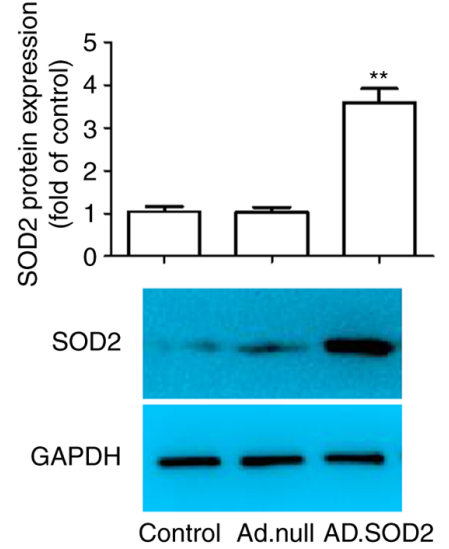

Figure 1. Flow cytometric identification of third-passage MSCs. Histograms show isotype controls (light red area) and cell surface marker expression (red line). The percentage of the expression level of the indicated markers was defined as the M1 region. Flow cytometric analysis identified that BM-MSCs were positive for (A) CD90 and (B) CD105, and negative for (C) CD34 and (D) CD45. Histograms show isotype controls (light red area) and cell surface marker expression (red line). The percentage of the expression level of the indicated markers was defined as the M1 region. (E) SOD2 mRNA expression was analyzed via reverse transcription-quantitative PCR. (F) SOD2 protein expression was determined using western blot analysis. Data are presented as the mean \pm SD from three independent experiments. ${ }^{* *} \mathrm{P}<0.01$ vs. Control. BM-MSCs, bone marrow-derived mesenchymal stem cells; SOD2, superoxide dismutase 2; Ad, adenovirus.
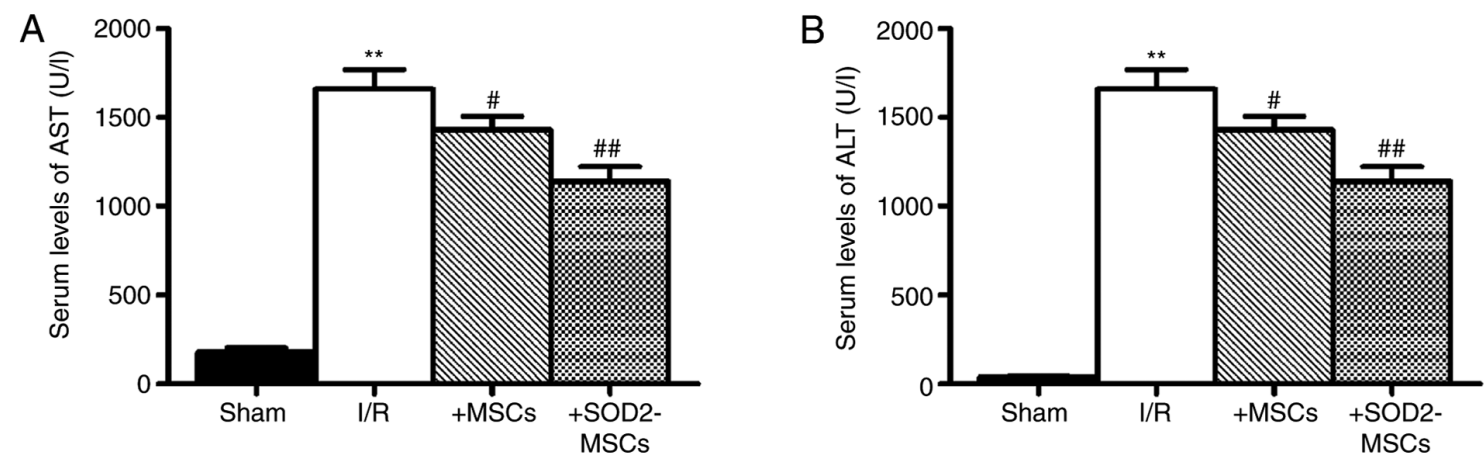

Figure 2. Effects of SOD2-overexpressing MSC transplantation on AST and ALT levels in the serum of rats with hepatic I/R injury. (A) AST and (B) ALT levels in the serum. Blood samples were collected after I/R and cell transplantation for ALT and AST analysis. Sham group, rats were subjected to laparotomy only; I/R group, rats were induced with I/R and received PBS; +MSCs, rats were induced with I/R and then transplanted with MSCs; and +SOD2-MSCs, rats were induced with $\mathrm{I} / \mathrm{R}$ and then transplanted with SOD2-overexpressing MSCs. $\mathrm{n}=8$ rats in each experimental group. Data are expressed as the mean \pm SD. ${ }^{* *} \mathrm{P}<0.01$ vs. sham; ${ }^{\#} \mathrm{P}<0.05$, ${ }^{\# /} \mathrm{P}<0.01$ vs. I/R. I/R, ischemia/reperfusion; SOD2, superoxide dismutase 2; MSCs, mesenchymal stem cells; ALT, alanine aminotransferase; AST, aspartate aminotransferase.

To verify SOD2 overexpression in BM-MSCs, Ad.SOD2 was transduced into BM-MSCs. After infection for $24 \mathrm{~h}$, the mRNA and protein expression levels of SOD2 were assessed. The results demonstrated that SOD2 mRNA and protein expression levels were significantly increased (Fig. 1E and F) in Ad.SOD2-transduced BM-MSCs compared with mock group or Ad.null-transduced BM-MSCs ( $\left.{ }^{* *} \mathrm{P}<0.01\right)$.

Effects of BM-MSCs overexpressing SOD2 on AST and ALT levels after HIRI. Elevated AST and ALT levels are important signs of severe liver injury (25). To determine the degree of I/R-induced hepatic injury in the liver tissues of the rat receiving BM-MSCs or SOD2-overexpressing BM-MSCs, ALT and AST levels were assessed after I/R and cell transplantation. Compared with the sham group, markers of liver damage, including ALT and AST, were significantly increased in I/R model rats $\left({ }^{* *} \mathrm{P}<0.01\right.$; Fig. $2 \mathrm{~A}$ and $\left.\mathrm{B}\right)$. However, there were significantly decreased levels of ALT and AST in the $\mathrm{I} / \mathrm{R}+$ MSCs and $\mathrm{I} / \mathrm{R}+\mathrm{SOD} 2-\mathrm{MSC}$ groups compared with the I/R group $\left({ }^{\#} \mathrm{P}<0.05\right)$. These findings suggested that the transplantation of SOD2-overexpressing BM-MSCs improved HIRI. 


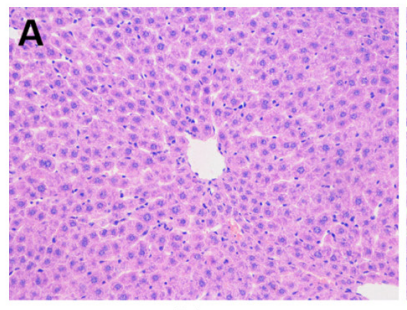

Sham

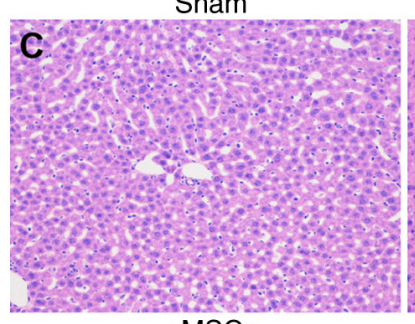

$+\mathrm{MSCS}$
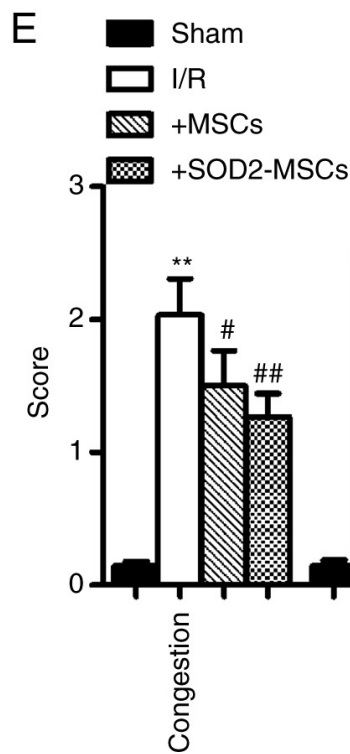

Figure 3. Histopathological analysis of liver tissues in rats. Liver tissue sections were stained with hematoxylin and eosin and scored according to the Suzuki Score System. Represented micrographs of liver tissue staining Representative micrographs of livers from the (A) sham, (B) I/R, (C) +MSCs and (D) +SOD2-MSCs groups. (E) Suzuki scores from each group. Sham group, rats were subjected to laparotomy only; I/R group, rats were induced with I/R and received PBS; +MSCs, rats were induced with I/R and then transplanted with MSCs; and +SOD2-MSCs, rats were induced with I/R and then transplanted with SOD2-overexpressing MSCs. $n=8$ rats in each experimental group. Data are expressed as the mean $\pm \mathrm{SD} .{ }^{* *} \mathrm{P}<0.01$ vs. sham; ${ }^{\#} \mathrm{P}<0.05,{ }^{\# \#} \mathrm{P}<0.01$ vs. I/R. I/R, ischemia/reperfusion; SOD2, superoxide dismutase 2; MSCs, mesenchymal stem cells.

BM-MSCs overexpressing SOD2 improve the histopathological changes of HIRI. To further confirm the role of SOD2 overexpression on HIRI in rats, the histopathology of livers harvested after $\mathrm{I} / \mathrm{R}$ induction and cell transplantation were examined. As shown in Fig. 3A-D, the pathological findings identified sinusoidal congestion, cytoplasmic vacuolization and necrosis in the I/R group, which are indicative of severe damage. Moreover, the results of the Suzuki scores indicated worse histopathology in the I/R group compared with the sham group $\left({ }^{* *} \mathrm{P}<0.01\right)$, whereas the scores indicated improved histopathology in the I/R + MSCs and I/R + SOD2-MSCs groups compared with the I/R group $\left({ }^{\#} \mathrm{P}<0.05\right.$; Fig. $\left.3 \mathrm{E}\right)$.
BM-MSCs overexpressing SOD2 attenuate the oxidative stress response in $I / R$ injury. The oxidative stress response is considered to be an important contributor to HIRI (26). Therefore, SOD, GSH-Px and MDA levels were assessed after HIRI induction. It was found that SOD and GSH-Px levels were markedly decreased, while the MDA level was notably increased in $\mathrm{I} / \mathrm{R}$ rats compared with the sham group $\left({ }^{*} \mathrm{P}<0.05\right.$; Fig. 4A-C). These findings indicated that elevated oxidative stress was an important contributor in liver injury. Moreover, in the I/R + MSCs and I/R + SOD2-MSCs groups, increased SOD and GSH-Px activities were observed, while MDA levels were decreased compared with the I/R group ( ${ }^{\mathrm{P}} \mathrm{P}<0.05$; Fig. 4A-C). In line with the results of the biochemical index, the production of reactive oxygen species (ROS) was detected in the liver tissues. The results demonstrated that an increased number of apoptotic cells was observed in the hepatic I/R group compared with the sham group, whereas the number of apoptotic cells was notably decreased in the I/R + MSCs and I/R + SOD2-MSCs groups compared with the I/R group (Fig. 4D-G). These findings suggested that transplanted SOD2-overexpressing BM-MSCs attenuated the oxidative stress in HIRI.

BM-MSCs overexpressing SOD2 inhibit cell apoptosis after HIRI. To investigate the effect of SOD2-overexpressing BM-MSCs against HIRI-related apoptosis, TUNEL staining was firstly utilized to examine the number of apoptotic cells and western blotting was used to detect the expression levels of Bcl-2, Bax and caspase-3. It was identified that hepatic I/R caused a markedly higher AI, suggesting that I/R resulted in hepatocyte apoptosis (Fig. 5A and B). Furthermore, the findings indicated a significant downregulation in Bcl-2 expression, and an upregulation in Bax and caspase-3 expression in the I/R group compared with the sham group $\left({ }^{*} \mathrm{P}<0.05\right.$; Fig. $\left.5 \mathrm{E}-\mathrm{H}\right)$. It was also found that these effects were partially reversed in the I/R + MSCs and I/R + SOD2-MSCs groups compared with the I/R group ( ${ }^{\#} \mathrm{P}<0.05$; Fig. $\left.5 \mathrm{C}-\mathrm{H}\right)$. Collectively, these results suggested that SOD2-overexpressing BM-MSCs exerted an inhibitory effect on cell apoptosis in HIRI.

\section{Discussion}

HIRI is a widespread clinical concern, which is common across multiple pathological conditions and liver surgical procedures, such as hemorrhagic shock, liver resection and liver transplantation (27). Hepatic I/R can lead to hepatic sinusoidal endothelial cell damage, Kupffer cell and leukocyte activation, leukocyte and platelet adhesion and microcirculation disorders, which directly affect the prognosis of the disease, the success rate of surgery and the survival rate of patients (28). The main mechanisms of HIRI are associated with intracellular calcium overload and oxygen free radicals (29). Thus, it is important for investigators to develop for novel strategies for hepatic I/R treatment.

BM-MSCs have a strong self-replication ability and multidifferentiation potential, which are based on their pluripotency and the secretion of beneficial molecules; therefore, these cells are widely used in basic and clinical trials $(6,13)$. It has been reported that BM-MSCs possess potent migratory and differentiation abilities under both physiological and pathological conditions, and may be cultured in vitro under specific 

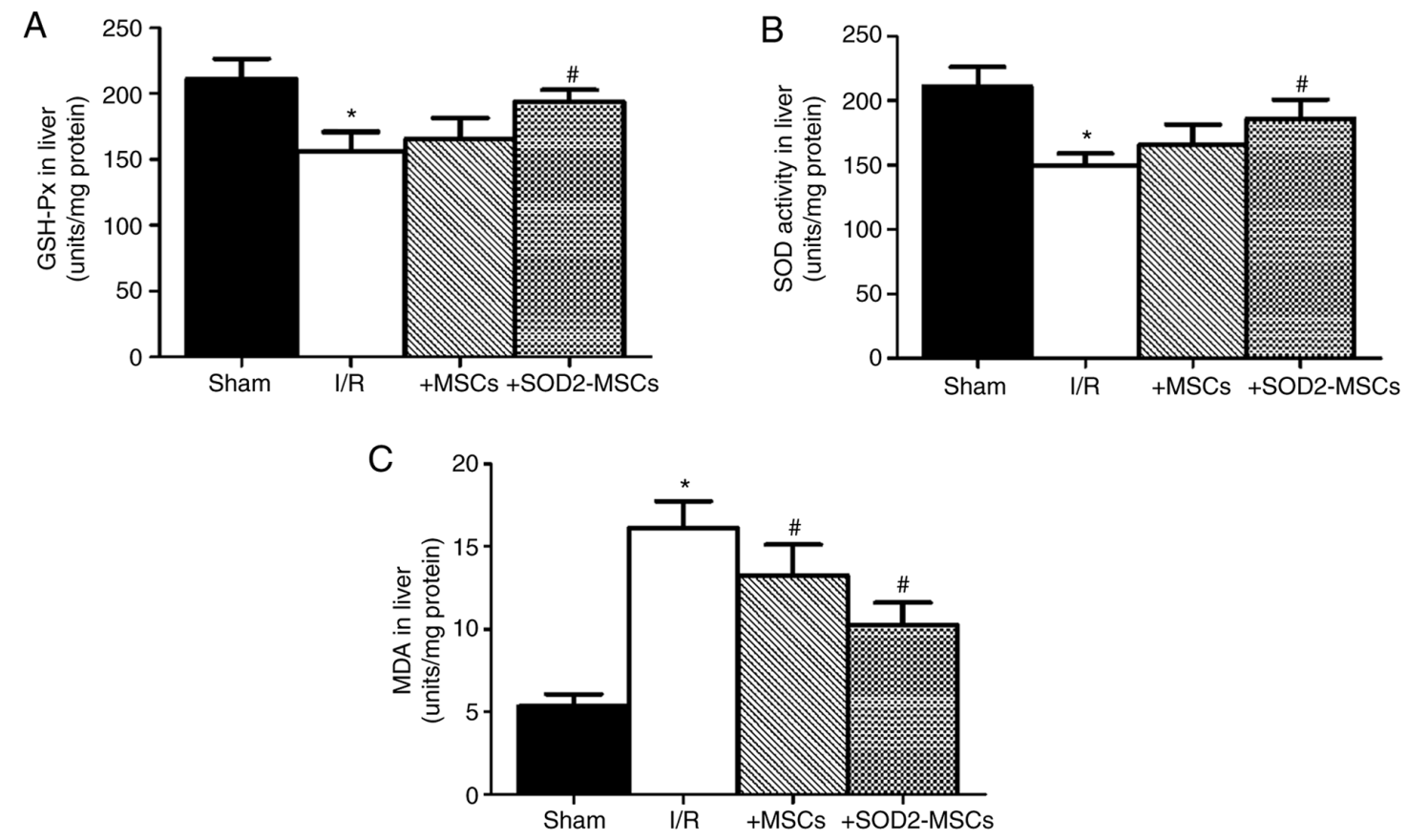

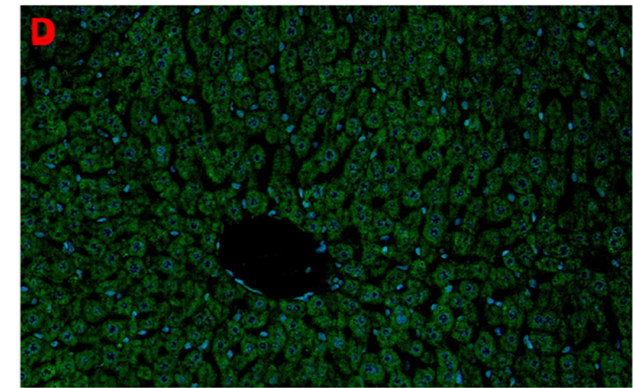

Sham

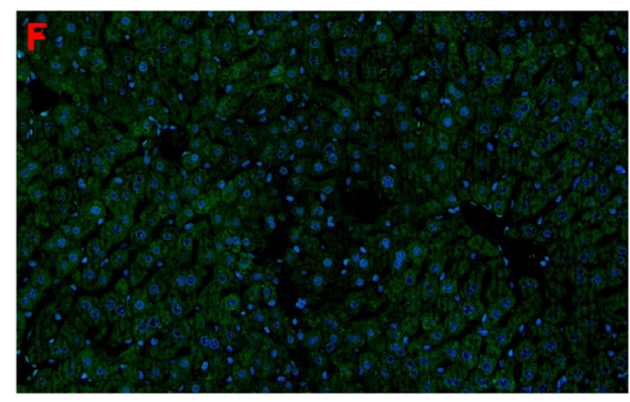

+ MSCs

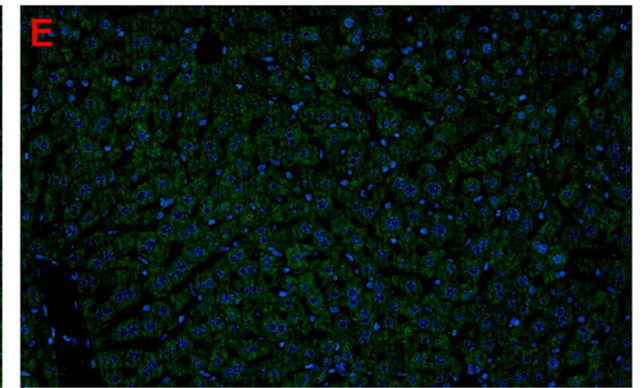

$\mathrm{I} / \mathrm{R}$

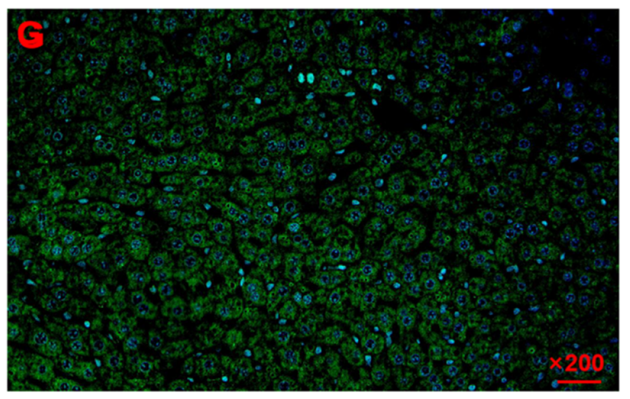

+ SOD2-MSCs

Figure 4. SOD2-overexpressing MSCs attenuate the oxidative stress response in hepatic I/R injury. (A) GSH-Px and (B) SOD activity; (C) MDA content. (D-G) ROS levels. Sham group, rats were subjected to laparotomy only; I/R group, rats were induced with I/R and received PBS; +MSCs, rats were induced with I/R and then transplanted with MSCs; and +SOD2-MSCs, rats were induced with I/R and then transplanted with SOD2-overexpressing MSCs. $\mathrm{n}=8$ rats in each experimental group. Data are expressed as the mean $\pm \mathrm{SD}$. ${ }^{*} \mathrm{P}<0.05$ vs. sham; ${ }^{*} \mathrm{P}<0.05$ vs. I/R. I/R, ischemia/reperfusion; SOD, superoxide dismutase; MSCs, mesenchymal stem cells; GSH-Px, glutathione peroxidase; MDA, malondialdehyde.

conditions to differentiate into cells derived from multiple germ layers, such as hepatocyte-like and adult cells $(30,31)$.

Accumulating evidence has indicated that nerve cells, adipocytes, osteoblasts and stem cell transplantation therapy are conducive to tissue damage repair and functional recovery (32-34). HIRI is a common pathological event during liver surgery, and is widely accepted as a common and inevitable complication during liver resection and liver transplantation, particularly in patients with end-stage liver disease (35). Moreover, the commonly used treatment methods for this include liver resection and liver transplantation, and thus, a method to reduce the $\mathrm{I} / \mathrm{R}$ injury of the liver during surgery will be of great significance to the clinical treatment of liver diseases (36). Previous studies have confirmed that BM-MSC transplantation exerts positive effects in treatment and has a protective function against liver tissue damage, and may be a potential intervention in the field of medicine and biology $(37,38)$. It has also been revealed that the overexpression of cytokines and growth factors targeting certain diseases may be effective strategies for dysfunction-targeted therapy (39). 


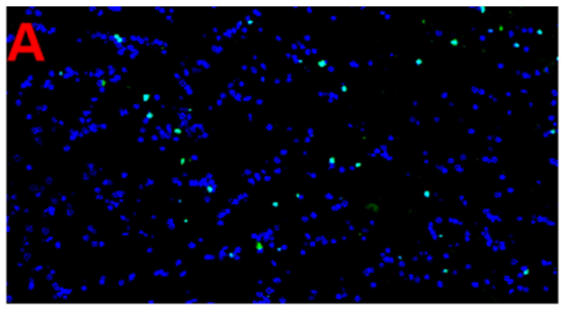

Sham

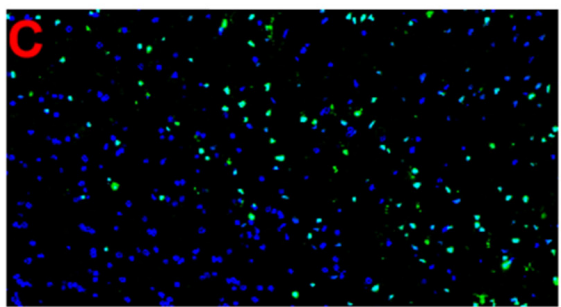

+MSCs

E

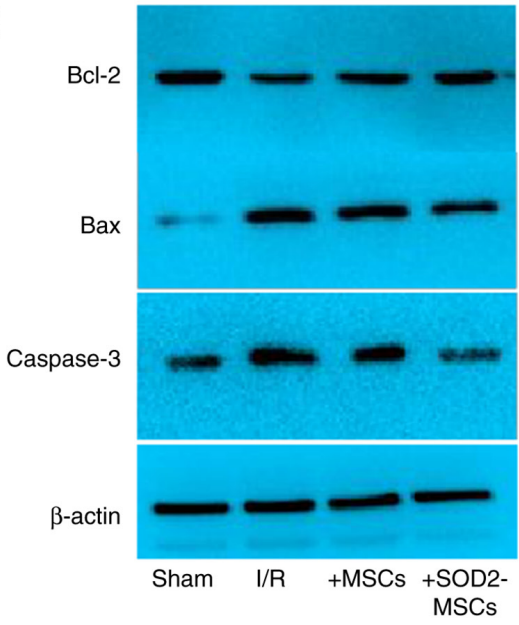

G

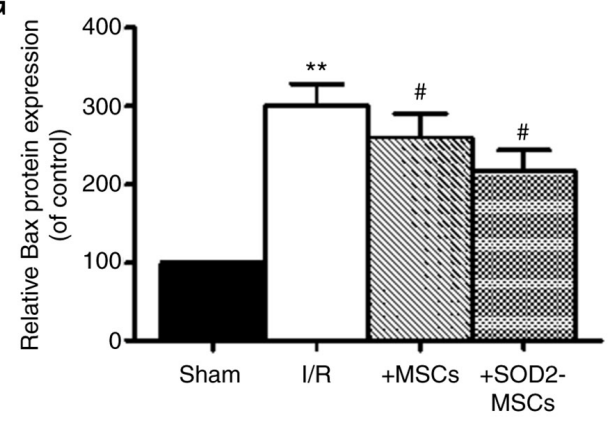

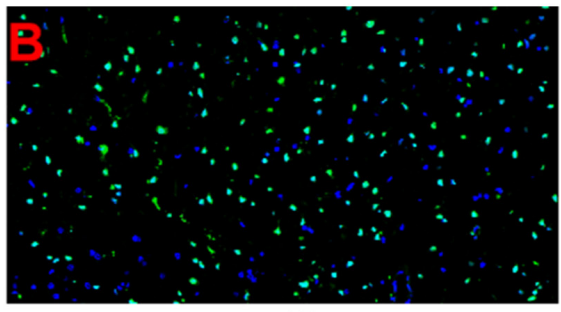

I/R

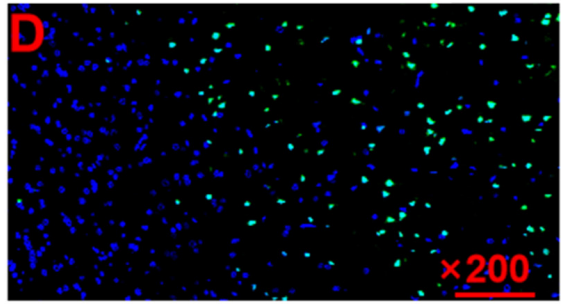

+SOD2-MSCs

$\mathrm{F}$

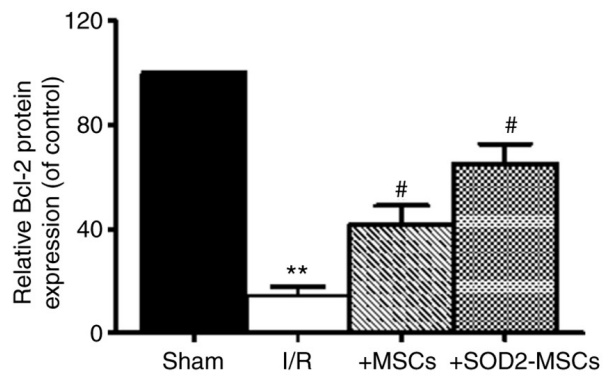

$\mathrm{H}$

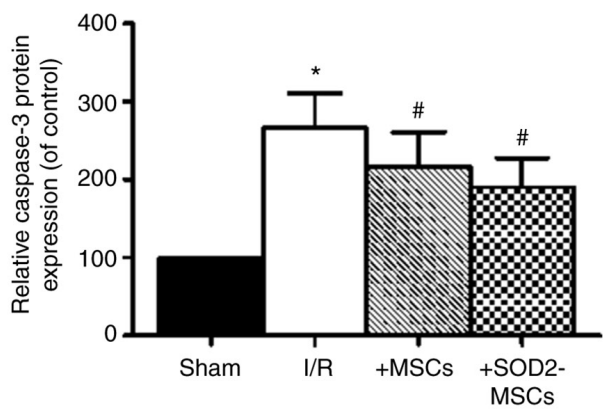

Figure 5. SOD2-overexpressing MSCs inhibit hepatocyte apoptosis. (A-D) TUNEL staining results. (E) Representative expression levels of Bcl-2, Bax and caspase-3 were analyzed via western blotting. $(\mathrm{F}-\mathrm{H})$ The relative expression levels of these proteins in relation to $\beta$-actin in each sample are presented. Sham group, rats were subjected to laparotomy only; I/R group, rats were induced with I/R and received PBS; +MSCs, rats were induced with I/R and then transplanted with MSCs; and +SOD2-MSCs, rats were induced with I/R and then transplanted with SOD2-overexpressing MSCs. n=8 rats in each experimental group. Data are expressed as the mean $\pm \mathrm{SD}$. ${ }^{*} \mathrm{P}<0.05,{ }^{* *} \mathrm{P}<0.01$ vs. sham; ${ }^{*} \mathrm{P}<0.05 \mathrm{vs}$. I/R. I/R, ischemia/reperfusion; SOD2, superoxide dismutase 2 ; MSCs, mesenchymal stem cells.

Therefore, the method of gene transfer allows BM-MSCs to express additional genes that are beneficial to treatment, and can enhance the efficacy of BM-MSC transplantation.

Intravenous administration of BM-MSCs has become a widely accepted treatment for decreasing intracellular calcium overload and oxygen free radicals, as well as for hepatoprotection following HIRI (13). The present study used the method of adenovirus transfection to overexpress the SOD2 gene in
MSCs, and RT-qPCR and western blotting demonstrated that SOD2 expression was upregulated in MSCs after adenovirus transfection, suggesting that SOD2 overexpression was successfully established. Moreover, SOD2 overexpression decreased oxidative stress and increased the expression levels of apoptosis-related genes.

Oxidative stress caused by hepatic $I / R$ is the main mechanism contributing to the deterioration of liver function 
induced by I/R (40). SODs are endogenous antioxidants that catalyze the conversion of $\mathrm{O} 2-$ to $\mathrm{H}_{2} \mathrm{O}_{2}$, and helps maintain the redox balance by diffusing the superoxide (41). SOD2, a member of the SOD family, is a mitochondrial antioxidant enzyme that scavenges superoxide radicals (42). The continuous expression of SOD2 is regulated by the cell oxygen concentration, and once hepatic hypoxic-ischemic injury occurs, SOD2 is activated (43). By regulating its downstream target genes, SOD2 participates in regulating anaerobic metabolism, cytokine production and regulating cell apoptosis (44). Therefore, SOD2 overexpression may be a promising novel intervention for the treatment of hepatic ischemia and hypoxic injury.

An increasing number of basic and clinical experiments have reported that SOD2 may serve a protective role in hepatocytes during HIRI (45). Reduction of SOD2 activity has been shown to decrease liver function induced by transient ischemia $(44,46)$. Moreover, overexpression of SOD2 can provide direct hepatic-protection via increasing levels of serum transaminases (including AST and ALT) and reversing pathological changes $(47,48)$. In animal models of HIRI, significant increases in liver SOD2 mRNA and protein expression levels have been observed $(47,48)$. These findings support the idea that SOD2-overexpressing BM-MSC transplantation may mitigate HIRI. In the present study, it was demonstrated that HIRI caused increases in the levels of AST and ALT, and SOD2-overexpressing BM-MSC transplantation obviously decreased the abnormal increases of these indicators, which in line with the reversal of pathological changes in HIRI.

In the current study, it was observed that SOD2overexpressing BM-MSCs exerted the protective effect of SOD2 against oxidative stress in rats subjected to hepatic I/R. Furthermore, SOD2 overexpression in BM-MSCs exerted protective effects in promoting liver function recovery and decreasing oxidative stress in a rat model of hepatic I/R. These effects were mediated via the amelioration of oxidative stress and apoptosis inhibition after BM-MSC transplantation. SODs are endogenous antioxidants, and their activities are associated with SOD2 levels. The present study demonstrated that SOD and GSH-Px activities were significantly increased, and MDA content was decreased after SOD2-overexpressing BM-MSC transplantation.

The concentration of lipid peroxides in the plasma during HIRI is closely associated with serum transaminase activity, and is parallel to the severity of liver morphological damage (49). The present findings indicated that I/R caused obvious necrosis and congestion in the injured liver lobules, accompanied by the presence of a large number of red blood cells in the venules and inflammatory cell infiltration. However, after SOD2-overexpressing BM-MSC transplantation, the liver tissue structure was partially restored to normal, although changes, such as dilated central veins, were still visible (50). These results suggested that MSC treatment regenerated damaged liver tissues subjected to I/R.

SOD2 exerts a hepatoprotective effect in HIRI, and several studies have reported that the increase in sirtuin (Sirt) 3 activity promotes the deacetylation of SOD2, which can decrease oxidative stress by eliminating ROS $(46,51)$. Sirt3 can stabilize hypoxia-inducible factor $1 \alpha(\mathrm{HIF}-1 \alpha)$, and HIF-1 $\alpha$ can activate the Sirt3 gene promoter, which leads to increased Sirt3 mRNA transcript synthesis and the inactivation of cyclophilin $\mathrm{D}$, thereby reducing the opening of the mitochondrial permeability transition pore channel. In turn, the extent of liver damage can be reduced (52). The present results demonstrated that SOD2 overexpression enhanced the therapeutic effect of BM-MSCs in HIRI, improved liver function and effectively reduced the degree of tissue injury. Compared with the BM-MSCs group and control group, liver tissue damage in the SOD2-MSCs group was significantly reduced. These results indicated that SOD2 overexpression could exert a positive synergistic effect with BM-MSCs in the treatment of liver injury.

However, the limitations of the current study have yet to be considered. Despite progress in revealing the ameliorative potentials of SOD2 overexpression in MSCs, the complete regulatory molecular targets of SOD2 overexpression require additional research. Moreover, the application of SOD2 overexpression in MSCs for the treatment of other liver I/R-related injuries, including liver transplantation, requires more rigorous and scientific verification, as well as subsequent clinical trials.

In conclusion, the present study demonstrated that BM-MSCs could be a potential new method for cell therapy, particularly in liver injury. At present, the mortality rate of liver injury is increasing worldwide annually, making the development of novel strategies for liver injury treatment highly important. The present study identified that transplantation of SOD2-overexpressing BM-MSCs may have the potential to ameliorate HIRI via the inhibition of oxidative stress and apoptosis.

\section{Acknowledgements}

The authors would like to thank the Laboratory Animal of Second Xiangya Hospital of Central South University for their technical assistance.

\section{Funding}

The present study was supported by the National Natural Science Foundation of China (grant nos. 81571784 and 30870695), the Provincial Natural Science Foundation of Hunan (grant nos. 2019JJ4044 and 2018JJ2578) and the Scientific Research Project of Hunan Health and Family Planning Commission (grant no. B20180048).

\section{Availability of data and materials}

All data generated or analyzed during this study are included in this published article.

\section{Authors' contributions}

QL, WZ and EX designed and performed experiments, and analyzed, interpreted and presented the results for group discussions. QL, WZ and EX confirm the authenticity of all the raw data. EX made substantial contributions to the conception of the study. All authors read and approved the final manuscript. 


\section{Ethics approval and consent to participate}

The protocol of the present study was approved by the Laboratory Animal Center, The Second Xiangya Hospital of Central South University (Changsha, China).

\section{Patient consent for publication}

Not applicable.

\section{Competing interests}

The authors declare that they have no competing interests.

\section{References}

1. Huang Y, Gao F, Zhang Y, Chen Y, Wang B, Zheng Y and Shi G: N-n-Butyl haloperidol iodide inhibits the augmented $\mathrm{Na}^{+} / \mathrm{Ca}^{2+}$ exchanger currents and L-type $\mathrm{Ca}^{2+}$ current induced by hypoxia/reoxygenation or $\mathrm{H} 2 \mathrm{O} 2$ in cardiomyocytes. Biochem Biophys Res Commun 421: 86-90, 2012.

2. Cannistrà M, Ruggiero M,Zullo A, Gallelli G, Serafini S, Maria M, Naso A, Grande R, Serra R and Nardo B: Hepatic ischemia reperfusion injury: A systematic review of literature and the role of current drugs and biomarkers. Int J Surg 33 (Suppl 1): S57-S70, 2016.

3. Hirakawa Y, Tsuchishima M, Fukumura A, Kinoshita K, Hayashi N, Saito T, George J, Toshikuni N, Ueda Y and Tsutsumi M: Recombinant thrombomodulin prevented hepatic ischemia-reperfusion injury by inhibiting high-mobility group box 1 in rats. Eur J Pharmacol 863: 172681, 2019.

4. Li J, Li RJ, Lv GY and Liu HQ: The mechanisms and strategies to protect from hepatic ischemia-reperfusion injury. Eur Rev Med Pharmacol Sci 19: 2036-2047, 2015.

5. Chao YH, Lin CW, Pan HH, Yang SF, Weng TF, Peng CT and $\mathrm{Wu} \mathrm{KH}$ : Increased apoptosis and peripheral blood mononuclear cell suppression of bone marrow mesenchymal stem cells in severe aplastic anemia. Pediatr Blood Cancer 65: e27247, 2018.

6. Rong Y, Liu W, Wang J, Fan J, Luo Y, Li L, Kong F, Chen J, Tang $\mathrm{P}$ and Cai W: Neural stem cell-derived small extracellular vesicles attenuate apoptosis and neuroinflammation after traumatic spinal cord injury by activating autophagy. Cell Death Dis 10: 340, 2019.

7. Zha JM, Li HS, Lin Q, Kuo WT, Jiang ZH, Tsai PY, Ding N, Wu J, Xu SF, Wang YT, et al: Interleukin 22 expands transit-amplifying cells while depleting $\mathrm{Lgr}^{+}$stem cells via inhibition of wnt and notch signaling. Cell Mol Gastroenterol Hepatol 7: 255-274, 2019.

8. Sun X, Luo L and Li J: LncRNA MALAT1 facilitates BM-MSCs differentiation into endothelial cells via targeting miR-206/VEGFA axis. Cell Cycle 19: 3018-3028, 2020

9. Zuo R, Liu M, Wang Y, Li J, Wang W, Wu J, Sun C, Li B, Wang Z, Lan W, et al: BM-MSC-derived exosomes alleviate radiation-induced bone loss by restoring the function of recipient BM-MSCs and activating Wnt/ $\beta$-catenin signaling. Stem Cell Res Ther 10: 30, 2019.

10. Tan YF, Tang L, OuYang WX, Jiang T, Zhang H and Li SJ: $\beta$-catenin-coordinated lncRNA MALAT1 up-regulation of ZEB-1 could enhance the telomerase activity in HGF-mediated differentiation of bone marrow mesenchymal stem cells into hepatocytes. Pathol Res Pract 215: 546-554, 2019.

11. Shay JW, Homma N, Zhou R, Naseer MI, Chaudhary AG, Al-Qahtan M, Hirokawa N, Goudarzi M, Fornace AJ Jr, Baeesa S, et al: Abstracts from the 3rd international genomic medicine conference (3rd IGMC 2015): Jeddah, kingdom of Saudi Arabia. 30 November-3 December 2015. BMC Genomics 17 (Suppl 6): S487, 2016.

12. Wang X, Wang S, Zhou Y, Obulkasim H, Zhang ZH, Dai B, Zhu W and Shi XL: BM-MSCs protect against liver ischemia/reperfusion injury via HO-1 mediated autophagy. Mol Med Rep 18: 2253-2262, 2018.

13. Yang B, Duan W, Wei L, Zhao Y, Han Z, Wang J, Wang M, Dai C, Zhang B, Chen D and Chen Z: Bone Marrow mesenchymal stem cell-derived hepatocyte-like cell exosomes reduce hepatic ischemia/reperfusion injury by enhancing autophagy. Stem Cells Dev 29: 372-379, 2020.
14. Zare MA, Zare A, Azarpira N and Pakbaz S: The protective effect of bone marrow-derived mesenchymal stem cells in liver ischemia/reperfusion injury via down-regulation of miR-370. Iran J Basic Med Sci 22: 683-689, 2019.

15. Zheng J, Chen L, Lu T, Zhang Y, Sui X, Li Y, Huang X, He L, Cai J, Zhou C, et al: MSCs ameliorate hepatocellular apoptosis mediated by PINK1-dependent mitophagy in liver ischemia/reperfusion injury through AMPK $\alpha$ activation. Cell Death Dis 11: 256, 2020.

16. Jin G, Qiu G, Wu D, Hu Y, Qiao P, Fan C and Gao F: Allogeneic bone marrow-derived mesenchymal stem cells attenuate hepatic ischemia-reperfusion injury by suppressing oxidative stress and inhibiting apoptosis in rats. Int J Mol Med 31: 1395-1401, 2013.

17. Chen P, Cui L, Chen G, You T, Li W, Zuo J, Wang C, Zhang W and Jiang C: The application of BMP-12-overexpressing mesenchymal stem cells loaded 3D-printed PLGA scaffolds in rabbit rotator cuff repair. Int J Biol Macromol 138: 79-88, 2019.

18. Shi X, Bai Y, Zhang G, Liu Y, Xiao H, Liu X and Zhang W: Effects of over-expression of SOD2 in bone marrow-derived mesenchymal stem cells on traumatic brain injury. Cell Tissue Res 372: 67-75, 2018.

19. Lee M, Song BR, Kim DH, Ha J, Lee M, Choi SJ, Oh W, Um S and Jin HJ: Up-regulation of superoxide dismutase 2 in 3D spheroid formation promotes therapeutic potency of human umbilical cord blood-derived mesenchymal stem cells. Antioxidants (Basel) 9: 66, 2020.

20. Mohr BJ, Fakoya FA, Hau J, Souilem O and Anestidou L: The Governance of Animal Care and Use for Scientific Purposes in Africa and the Middle East. ILAR J 57: 333-346, 2016.

21. Krüger-Haag A, Lehmann C, Schmidt E, Sonntag F, Hörer M and Kochanek S: Evaluation of life cycle defective adenovirus mutants for production of adeno-associated virus vectors. J Gene Med 21: e3094, 2019.

22. Kronenburg A, Bulder MMM, Bokkers RPH, Hartkamp NS, Hendrikse J, Vonken EJ, Kappelle LJ, van der Zwan A, Klijn CJM and Braun KPJ: Cerebrovascular reactivity measured with ASL perfusion MRI, Ivy sign, and regional tissue vascularization in moyamoya. World Neurosurg 125: e639-e650, 2019.

23. Livak KJ and Schmittgen TD: Analysis of relative gene expression data using real-time quantitative PCR and the 2(-Delta Delta C(T)) method. Methods 25: 402-408, 2001.

24. Lv FJ, Tuan RS, Cheung KM and Leung VY: Concise review: The surface markers and identity of human mesenchymal stem cells. Stem Cells 32: 1408-1419, 2014.

25. Xu L, Yu Y, Sang R, Li J, Ge B and Zhang X: Protective effects of taraxasterol against ethanol-induced liver injury by regulating CYP2E1/Nrf2/HO-1 and NF-kappaB signaling pathways in mice. Oxid Med Cell Longev 2018: 8284107, 2018.

26. Yuan R, Tao X, Liang S, Pan Y, He L, Sun J, Wenbo J, Li X, Chen J and Wang C: Protective effect of acidic polysaccharide from Schisandra chinensis on acute ethanol-induced liver injury through reducing CYP2E1-dependent oxidative stress. Biomed Pharmacother 99: 537-542, 2018.

27. Zhang M, Yang D, Gong X, Ge P, Dai J, Lin L and Zhang L: Protective benefits of AMP-activated protein kinase in hepatic ischemia-reperfusion injury. Am J Transl Res 9: 823-829, 2017.

28. Peralta C, Rull R, Rimola A, Deulofeu R, Roselló-Catafau J, Gelpí E and Rodés J: Endogenous nitric oxide and exogenous nitric oxide supplementation in hepatic ischemia-reperfusion injury in the rat. Transplantation 71: 529-536, 2001.

29. Hataji K, Watanabe T, Oowada S, Nagaya M, Kamibayashi M, Murakami E, Kawakami H, Ishiuchi A, Kumai T, Nakano H, et al: Effects of a calcium-channel blocker (CV159) on hepatic ischemia/reperfusion injury in rats: Evaluation with selective $\mathrm{NO} / \mathrm{pO} 2$ electrodes and an electron paramagnetic resonance spin-trapping method. Biol Pharm Bull 33: 77-83, 2010.

30. Charbord P: Bone marrow mesenchymal stem cells: Historical overview and concepts. Hum Gene Ther 21: 1045-1056, 2010.

31. Zhou W, Lin J, Zhao K, Jin K, He Q, Hu Y, Feng G, Cai Y, Xia C, Liu $\mathrm{H}$, et al: Single-cell profiles and clinically useful properties of human mesenchymal stem cells of adipose and bone marrow origin. Am J Sports Med 47: 1722-1733, 2019.

32. Kassis I, Vaknin-Dembinsky A and Karussis D: Bone marrow mesenchymal stem cells: Agents of immunomodulation and neuroprotection. Curr Stem Cell Res Ther 6: 63-68, 2011.

33. Miao C, Lei M, Hu W, Han S and Wang Q: A brief review: The therapeutic potential of bone marrow mesenchymal stem cells in myocardial infarction. Stem Cell Res Ther 8: 242, 2017.

34. Yang W and Ma B: A mini-review: The therapeutic potential of bone marrow mesenchymal stem cells and relevant signaling cascades. Curr Stem Cell Res Ther 14: 214-218, 2019. 
35. Yang C, Yang HJ, Deng SP and Zhang Y: Current status of ex-vivo liver resection and autologous liver transplantation for end-stage hepatic alveolar echinococcosis. Ann Palliat Med 9: 2271-2278, 2020.

36. Konishi $\mathrm{T}$ and Lentsch AB: Hepatic Ischemia/reperfusion: Mechanisms of tissue injury, repair, and regeneration. Gene Expr 17: 277-287, 2017.

37. Mohamed HE, Elswefy SE, Rashed LA, Younis NN, Shaheen MA and Ghanim AM: Bone marrow-derived mesenchymal stem cells effectively regenerate fibrotic liver in bile duct ligation rat model. Exp Biol Med (Maywood) 241: 581-591, 2016.

38. Souza MC, Silva JD, Pádua TA, Torres ND, Antunes MA, Xisto DG, Abreu TP, Capelozzi VL, Morales MM, Sá Pinheiro AA, et al: Mesenchymal stromal cell therapy attenuated lung and kidney injury but not brain damage in experimental cerebral malaria. Stem Cell Res Ther 6: 102, 2015.

39. Jain R, Dey B, Khera A, Srivastav P, Gupta UD, Katoch VM, Ramanathan VD and Tyagi AK: Over-expression of superoxide dismutase obliterates the protective effect of BCG against tuberculosis by modulating innate and adaptive immune responses. Vaccine 29: 8118-8125, 2011.

40. Du Y, Qian B, Gao L, Tan P, Chen H, Wang A, Zheng T, Pu S, $\mathrm{Xia} \mathrm{X}$ and $\mathrm{Fu} \mathrm{W}$ : Aloin preconditioning attenuates hepatic ischemia/reperfusion injury via inhibiting TLR4/MyD88/NF- $\kappa \mathrm{B}$ signal pathway in vivo and in vitro. Oxid Med Cell Longev 2019: 3765898, 2019.

41. Marampon F, Codenotti S, Megiorni F, Del Fattore A, Camero S, Gravina GL, Festuccia C, Musio D, De Felice F, Nardone V, et al: NRF2 orchestrates the redox regulation induced by radiation therapy, sustaining embryonal and alveolar rhabdomyosarcoma cells radioresistance. J Cancer Res Clin Oncol 145: 881-893, 2019.

42. Zelko IN, Mariani TJ and Folz RJ: Superoxide dismutase multigene family: A comparison of the CuZn-SOD (SOD1), Mn-SOD (SOD2), and EC-SOD (SOD3) gene structures, evolution, and expression. Free Radical Biol Med 33: 337-349, 2002.

43. Katwal G, Baral D, Fan X, Weiyang H, Zhang X, Ling L, Xiong Y, Ye Q and Wang Y: SIRT3 a major player in attenuation of hepatic ischemia-reperfusion injury by reducing ROS via its downstream mediators: SOD2, CYP-D, and HIF-1 $\alpha$. Oxid Med Cell Longev 2018: 2976957, 2018.

44. Miyauchi T, Uchida Y, Kadono K, Hirao H, Kawasoe J, Watanabe T, Ueda S, Jobara K, Kaido T, Okajima H, et al: Preventive effect of antioxidative nutrient-rich enteral diet against liver ischemia and reperfusion injury. JPEN J Parenter Enteral Nutr 43: 133-144, 2019.
45. Elias-Miró M, Mendes-Braz M, Cereijo R, Villarroya F, Jiménez-Castro MB, Gracia-Sancho J, Guixé-Muntet S, Massip-Salcedo M, Domingo JC, Bermudo R, et al: Resistin and visfatin in steatotic and non-steatotic livers in the setting of partial hepatectomy under ischemia-reperfusion. J Hepatol 60: 87-95, 2014

46. Katwal G, Baral D, Fan X, Weiyang H, Zhang X, Ling L, Xiong Y, Ye Q and Wang Y: SIRT3 a major player in attenuation of hepatic ischemia-reperfusion injury by reducing ROS via its downstream mediators: SOD2, CYP-D, and HIF-1 $\alpha$. Oxid Med Cell Longev 2018: 2976957, 2018.

47. Wheeler MD, Katuna M, Smutney OM, Froh M, Dikalova A, Mason RP, Samulski RJ and Thurman RG: Comparison of the effect of adenoviral delivery of three superoxide dismutase genes against hepatic ischemia-reperfusion injury. Hum Gene Ther 12: 2167-2177, 2001.

48. Wheeler MD, Nakagami M, Bradford BU, Uesugi T, Mason RP, Connor HD, Dikalova A, Kadiiska M and Thurman RG: Overexpression of manganese superoxide dismutase prevents alcohol-induced liver injury in the rat. J Biol Chem 276: 36664-36672, 2001.

49. Rodríguez-Reynoso S, Leal-Cortés C, Portilla-de Buen E and López-De la Torre SP: Ischemic preconditioning preserves liver energy charge and function on hepatic ischemia/reperfusion injury in rats. Arch Med Res 49: 373-380, 2018.

50. Wang X, Wang S, Zhou Y, Obulkasim H, Zhang ZH, Dai B, Zhu W and Shi XL: BM-MSCs protect against liver ischemia/reperfusion injury via HO-1 mediated autophagy. Mol Med Rep 18: 2253-2262, 2018.

51. Li DP, Chen YL, Jiang HY, Chen Y, Zeng XQ, Xu LL, Ye Y, Ke CQ, Lin G, Wang JY and Gao H: Phosphocreatine attenuates Gynura segetum-induced hepatocyte apoptosis via a SIRT3-SOD2-mitochondrial reactive oxygen species pathway. Drug Des Devel Ther 13: 2081-2096, 2019.

52. Wang Q, Wei S, Li L, Qiu J, Zhou S, Shi C, Shi Y, Zhou H and Lu L: TGR5 deficiency aggravates hepatic ischemic/reperfusion injury via inhibiting SIRT3/FOXO3/HIF-1 pathway. Cell Death Discov 6: 116, 2020.

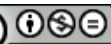

This work is licensed under a Creative Commons Attribution-NonCommercial-NoDerivatives 4.0 International (CC BY-NC-ND 4.0) License. 